\title{
Intracystic papillary neoplasm of the gallbladder concomitant with xanthogranulomatous cholecystitis: a case report
}

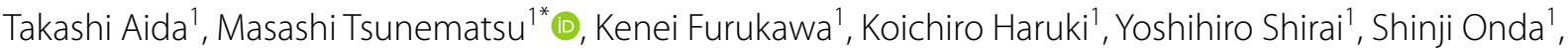 \\ Yoichi Toyama' ${ }^{1}$ Kazutaka Gomisawa², Hiroyuki Takahashi ${ }^{2}$ and Toru Ikegami ${ }^{1}$
}

\begin{abstract}
Background: The intracystic papillary neoplasm (ICPN) is a newly established disease concept. It has been regarded as a preinvasive neoplastic lesion, similar to intraductal papillary mucinous neoplasm of the pancreas. Limited information is available on the clinical and imaging features of ICPN.

Case presentation: A 65-year-old woman was referred to our hospital for assessment of a gallbladder tumor. Contrast-enhanced computed tomography showed a papillary tumor in the fundus of the gallbladder with irregular thickening of the gallbladder wall that spread into the cystic duct. The boundary between the tumor and liver was unclear. The patient was diagnosed with gallbladder cancer with liver invasion. We performed extended cholecystectomy with liver bed resection after confirming the absence of cancer cells in the resection margin of the cystic duct. After pathological examination, the tumor was diagnosed as an ICPN with xanthogranulomatous cholecystitis. The patient was discharged on postoperative day 8 with no complications.
\end{abstract}

Conclusions: We have described a rare case of ICPN concomitant with xanthogranulomatous cholecystitis. Clinicians should include ICPN as a differential diagnosis in patients with a papillary or polypoid tumor in the gallbladder.

Keywords: Intracystic papillary neoplasm, Gallbladder, Xanthogranulomatous cholecystitis

\section{Background}

An intracystic papillary neoplasm (ICPN) is classified as a premalignant gallbladder lesion in the 2018 classification of the World Health Organization [1]. However, the morphological characteristics of ICPN remain unclear because of its rarity. Additionally, it is sometimes difficult to discriminate gallbladder carcinomas from cholecystitis during the preoperative period [2].

\footnotetext{
*Correspondence: tsunematsu@jikei.ac.jp

1 Division of Hepatobiliary and Pancreatic Surgery, Department of Surgery, The Jikei University School of Medicine, 3-25-8,

Nishi-Shinbashi, Minato-ku, Tokyo 105-8461, Japan

Full list of author information is available at the end of the article
}

We herein report a case of ICPN concomitant with xanthogranulomatous cholecystitis (XGC) and review previously published reports of ICPN.

\section{Case presentation}

A 65-year-old woman was referred to our hospital for assessment of a gallbladder tumor that had been detected by abdominal ultrasonography during a medical checkup. She had no symptoms. Contrast-enhanced computed tomography revealed a papillary lesion $(25 \mathrm{~mm}$ in diameter) in the fundus of the gallbladder with irregular thickening of the gallbladder wall. The boundary between the tumor and liver was unclear, and the wall thickening extended from the fundus to the cystic duct (Fig. 1A-C). 


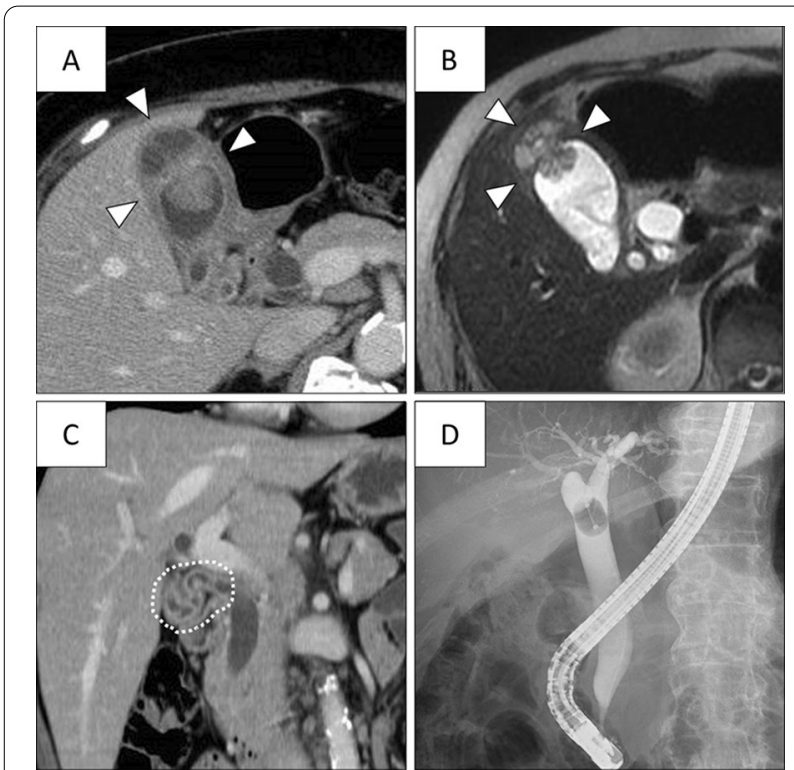

Fig. 1 Enhanced computed tomography and T2-weighted magnetic resonance imaging. A, B A papillary or polypoid tumor was present in the gallbladder. $\mathbf{C}$ The gallbladder wall was generally thickened, and the thickening continued to the cystic duct. D Endoscopic retrograde cholangiography revealed a filling defect in the cystic duct

Endoscopic retrograde cholangiography showed a complete filling defect in the cystic duct (Fig. 1D). Blood test showed inflammatory maker did not elevate. Serum carcinoembryonic antigen level was $2.1 \mathrm{ng} / \mathrm{ml}$, and serum carbohydrate antigen 19-9 level was $24 \mathrm{U} / \mathrm{ml}$. According to these findings, we diagnosed the tumor as gallbladder cancer invading the liver and cystic duct, and we decided to perform extended cholecystectomy.

There were no adhesions around the gallbladder, while the gallbladder wall was thickened. The hard tumor was detected at the fundus. Intraoperative ultrasonography revealed the tumor did not invade liver obviously. Inflammation changes were seen around the neck and Calot triangle. After ligation and dissection of the cystic duct at the junction, the intraoperative frozen section of the cystic duct stump and 2 sentinel lymph nodes were negative for malignancy; therefore, we performed extended cholecystectomy with liver bed resection.

Macroscopic examination of the resected specimen showed a superficially spreading papillary tumor with thick mucus on its surface (Fig. 2A, B). The gallbladder wall was diffusely thickened. Pathological examination revealed that the gallbladder neoplasm was composed of atypical cells arranged in a papillary architecture along with the development of fibrovascular stalks. These tall columnar cells contained large amounts of mucus (Fig. 2C). Ovarian-like stroma was not detected. The nucleolus body was remarkable, but the tumor was

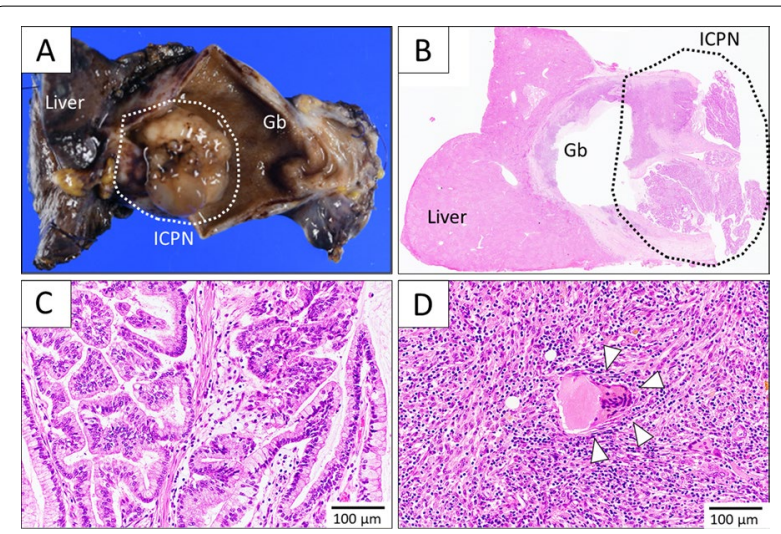

Fig. 2 Pathological findings. A Gross inspection of the resected specimens revealed that the mass had extroversive development from the mucosa of the gallbladder and contained some mucinous cysts. The mucus adhered to the surface of tumors. B-D Hematoxylin and eosin-stained histological sections. B Magnification $\times 40$. C Magnification $\times$ 200. D Magnification $\times 200$. The papillary growth was composed of atypical cells along with the development of fibrovascular stalks. Some of these tall columnar cells contained rich mucus. There was no evidence of tumor invasion. The gallbladder wall was highly thickened by the presence of severe inflammatory lesions containing lymphocytes and multinucleated giant cells, which were considered to indicate chronic granulomatous changes in the wall. The histological diagnosis was intracystic papillary neoplasm with xanthogranulomatous cholecystitis

noninvasive and showed no evidence of lymph node metastasis. These pathological findings were compatible with ICPN. In immunohistochemical staining, MUC5AC and MUC6 were strongly positive. CK7 and MUC1 were also positive, but not CK20, MUC2, estrogen receptor, and progesterone receptor. Immunohistochemistry indicated that ICPN was predominantly gastric type, with focal pancreatobiliary type. In addition, many lymphocytes and multinucleated giant cells had infiltrated the thickened gallbladder wall with prominent Rokitansky-Aschoff sinuses. These finding were especially seen at the fundus and were indicative of chronic granulomatous changes within the fundus (Fig. 2D). Based on these pathological findings, we diagnosed the tumor as an ICPN concomitant with XGC.

The patient was discharged on postoperative day 8 with no complications. She was clinically well with no evidence of recurrence at 3 months after resection.

\section{Discussion}

The ICPN is a relatively new disease concept that was first described in the 2010 World Health Organization classification [3]. It has been regarded as a counterpart disease of intraductal papillary neoplasm of the bile duct and intraductal papillary mucinous neoplasm of the pancreas. ICPNs more often occur in women older than 60 years, 
Table 1 Clinical characteristics of patients with ICPN

\begin{tabular}{|c|c|c|c|c|c|c|c|c|}
\hline Case & Age & Sex & Symptoms & Imaging & Diagnosis & Surgery & Outcome & References \\
\hline 1 & 48 & $\mathrm{~F}$ & No & Cystic tumor & - & LC & n.d & [7] \\
\hline 2 & 71 & $\mathrm{~F}$ & Epigastric pain & Wall thickening & - & LC & Alive 30 months & [8] \\
\hline 3 & 86 & $\mathrm{~F}$ & Jaundice & Papillary tumor, wall thickening & ICPN & LC & n.d & [9] \\
\hline 4 & 58 & $\mathrm{~F}$ & Fever & Papillary tumor & ICPN & SSPPD & Alive 6 months & [10] \\
\hline 5 & 71 & M & No & Papillary tumor & ICPN & LC & n.d & [11] \\
\hline 6 & 78 & $\mathrm{~F}$ & Epigastric pain & Papillary tumor & Gallbladder tumor & LC & Alive 12 months & [5] \\
\hline 7 & 64 & M & Epigastric pain & Cystic tumor & Gallbladder tumor & LC & n.d & [12] \\
\hline 8 & 54 & $\mathrm{~F}$ & Epigastric pain & Papillary and nodular tumor & Gallbladder tumor & ExC & Alive 2 months & [13] \\
\hline 9 & 74 & $\mathrm{~F}$ & No & Papillary tumor & $\mathrm{GbC}$ & ExC & n.d & [14] \\
\hline 10 & 61 & $\mathrm{~F}$ & No & Papillary tumor & $\mathrm{GbC}$ & ExC & n.d & [14] \\
\hline 11 & 83 & M & No & Papillary tumor & $\mathrm{GbC}$ & ExC & n.d & [14] \\
\hline 12 & 65 & $\mathrm{~F}$ & No & Papillary tumor, Wall thickening & $\mathrm{GbC}$ & ExC & Alive 2 months & Present case \\
\hline
\end{tabular}

ExC extended cholecystectomy, $F$ female, GbC gallbladder cancer, ICPN intracystic papillary neoplasm, LC laparoscopic cholecystectomy, $M$ male, $n . d$ no data, SSPPD subtotal stomach preserving pancreaticoduodenectomy

and their incidence in women is twice as high as that in men [1]. Almost $50 \%$ of patients with ICPNs develop abdominal pain in the peripheral aspect of the upper quadrant, while the remaining $50 \%$ are asymptomatic and incidentally found to have a tumor, as in our case [4]. ICPNs are considered to be precancerous lesions. The prognosis of ICPNs is much better than that of invasive gallbladder carcinomas. In fact, the 3-year survival rate of patients with noninvasive and invasive ICPN is $90 \%$ and $60 \%$, respectively [4].

The pathological characteristics of ICPN are macroscopic papillary growth within the gallbladder, regardless of mucin production, and the microscopic presence of intraductal papillary growth with delicate fibrovascular stalks [5]. Therefore, ICPNs can be diagnosed only after analysis of resected specimens, and they are found in $<0.5 \%$ of cholecystectomies $[4,6]$. Because of the limited number of case reports, the main characteristics of ICPNs remain unclear. We searched PubMed using the keywords "gallbladder" and "intracystic papillary neoplasm" or "intracholecystic papillary neoplasm" from 2010 to 2021 and found 11 case reports of ICPNs [5, 714]. We summarized 12 cases (all 11 previously published cases in addition to the present case) in Table 1, focusing on the imaging features and preoperative diagnoses.

Of these 12 cases, papillary or polypoid lesions were present in the gallbladder in 9 cases (75\%). However, gallbladder wall thickening was uncommon. ICPN could be diagnosed preoperatively in only three cases. Two patients underwent biopsy using peroral cholangioscopy, and another patient underwent cytology using endoscopic naso-gallbladder drainage. These results suggest that biopsy may help to achieve a definitive diagnosis in patients with papillary or polypoid lesions. One patient with a preoperative diagnosis of ICPN located only in the gallbladder underwent laparoscopic cholecystectomy without extended resection.

In our case, because computed tomography and magnetic resonance imaging showed not only a papillary tumor, but also irregular wall thickening, the possibility of gallbladder carcinoma needed to be considered. The radiological findings of XGC, such as the various patterns of wall thickening, resemble those of gallbladder carcinomas [15]. Therefore, the presence of XGC made a precise diagnosis difficult to achieve in the current case. Moreover, the mucus produced by the ICPN obstructed the thickened and narrowed gallbladder duct, preventing cytology and biopsy via the duct during endoscopic retrograde cholangiography. Given that intraoperative frozen section analysis is useful in distinguishing XGC and gallbladder carcinomas [16], careful intraoperative diagnosis may help us to choose the optimal operative procedure.

\section{Conclusions}

We have herein reported a rare case of ICPN with XGC. Clinicians should include ICPN as a differential diagnosis in patients with a papillary or polypoid tumor in the gallbladder, keeping in mind that biopsy may allow for a definitive diagnosis.

\section{Abbreviations}

ICPN: Intracystic papillary neoplasm; XGC: Xanthogranulomatous cholecystitis.

\section{Acknowledgements}

This research was supported by The Jikei University Research Fund "2021003SR". The authors thank Angela Morben, DVM, ELS, from Edanz (https://jp. edanz.com/ac) for editing a draft of this manuscript. 


\section{Authors' contributions}

TA, MT, and KH for drafting the manuscript. KF, YS, SO, YT, KG, and HT for acquisition of the data and critical revision. TI for final approval of the manuscript. All authors are in agreement with the content of the manuscript. The authors declare that they have no competing interest regarding this manuscript. All authors read and approved the final manuscript.

\section{Funding}

None.

\section{Availability of data and materials}

The data that support the findings of this study are available from the corresponding author upon reasonable request.

\section{Declarations}

\section{Ethics approval and consent to participate}

This retrospective study meeting the ethical standards of the World Medical Association Declaration of Helsinki was approved by The Jikei University School of Medicine [27-177(8062)].

\section{Consent for publication}

The patient has given consent for the publication of images.

\section{Competing interests}

The authors declare that they have no competing interests.

\section{Author details}

'Division of Hepatobiliary and Pancreatic Surgery, Department of Surgery, The Jikei University School of Medicine, 3-25-8, Nishi-Shinbashi, Minato-ku, Tokyo 105-8461, Japan. ${ }^{2}$ Department of Pathology, The Jikei University School of Medicine, Tokyo, Japan.

Received: 2 September 2021 Accepted: 15 October 2021

Published online: 24 October 2021

\section{References}

1. WHO Classification of Tumours Editorial Board Digestive system tumours Lyon (France) International Agency for Research on Cancer. WHO classification of tumours series. (5th ed) 2019;1.

2. Ewelukwa O, Ali O, Akram S. Xanthogranulomatous cholecystitis mimicking gallbladder cancer. BMJ Case Rep. 2013;10:1136.

3. Albores-Saavedra J, Adsay NV, Crawford JM, et al. Carcinoma of the gallbladder and extrahepatic ducts. In: Bosman FT, Carneiro F, Hruban RH, et al., editors. WHO classification of tumours of the digestive system. 4th ed. Lyon: International Agency for Research on Cancer; 2010. p. 266-73.
4. Adsay V, Jang KT, Roa JC, Dursun N, Ohike N, Bagci P, et al. Intracholecystic papillary-tubular neoplasms (ICPN) of the gallbladder (neoplastic polyps, adenomas, and papillary neoplasms that are $\geq 1.0 \mathrm{~cm}$ ): clinicopathologic and immunohistochemical analysis of 123 cases. Am J Surg Pathol. 2012;36:1279-301.

5. Oba T, Sato N, Tamura T, Komeda M, Adachi Y, Matsuyama A, et al. A case of gallbladder adenocarcinoma arising in association with intracystic papillary neoplasm (ICPN) with abundant mucin production. Clin J Gastroenterol. 2021;14:319-24.

6. Argon A, Barbet FY, Nart D. The relationship between intracholecystic papillary-tubular neoplasms and invasive carcinomas of gallbladder. Int J Surg Pathol. 2016;24:504-11.

7. Ismail R, Saeed Bamashmos A, Cohen PJ, Pucar D. Intracystic papillary neoplasm of gallbladder mimicking metastatic malignancy on PET/CT. Clin Nucl Med. 2021;46:e363-4.

8. Ana L, Julio C, Carlos D, Jorge P, Maria TC, Carlos C. Low-grade dysplastic intracholecystic papillary neoplasia. Am J Case Rep. 2021;22:e929788.

9. Kuniyoshi N, Imazu H, Hayama J, Nomura S, Kagawa A, Hamana S, et al. Intracholecystic papillary neoplasm of the gallbladder preoperative diagnosed by endoscopic ultrasonography and peroral cholangioscopy. ACG Case Rep J. 2021;8:e00574.

10. Yokode M, Hanada K, Shimizu A, Minami T, Hirohata R, Amano H, et al. Intracholecystic papillary neoplasm of the gallbladder protruding into the common bile duct. Mole and Clin Onc. 2019;11:488-92.

11. Hara A, Kamata K, Takenaka M, Chikugo T, Kudo M. Intracystic papillary neoplasm preoperatively diagnosed by high-quality cytology derived from endoscopic nasogallbladder drainage. Gastrointestinal Endscopy. 2019;89:1257-9.

12. Sato R, Ando T, Hiroo T, Rikiyama T, Furukawa T, Ebina N. Intracystic papillary neoplasm with an associated mucinous adenocarcinoma arising in Rokitansky-Aschoff sinus of the gallbladder. Surg Case Rep. 2016;2:62.

13. Meguro Y, Fukushima N, Koizumi M, Kasahara N, Hydo M, Morishima K, et al. A case of mixed adenoneuroendocrine carcinoma of the gallbladder arising from an intracystic papillary neoplasm associated with pancreaticobiliary maljunction. Pathol Int. 2014;64:465-71.

14. Mizoguchi N, Munechika J, Takeyama N, Ohgiya Y, Ohike N, Abe R, et al. Three cases of intracystic papillary neoplasm of gallbladder. Abdom Radiol. 2018:43:1535-9.

15. Shetty GS, Abbey P, Prabhu SM, Narula MK, Anand R. Xanthogranulomatous cholecystitis: sonographic and CT features and differentiation from gallbladder carcinoma: a pictorial essay. Jpn J Radiol. 2012;30:480-5.

16. Makimoto S, Takami T, Hatano K, Kataoka N, Yamaguchi T, Tomita M, et al. Xanthogranulomatous cholecystitis: a review of 31 patients. Surg Endosc. 2021;35:3874-80.

\section{Publisher's Note}

Springer Nature remains neutral with regard to jurisdictional claims in published maps and institutional affiliations.

\section{Submit your manuscript to a SpringerOpen ${ }^{\circ}$ journal and benefit from:}

- Convenient online submission

- Rigorous peer review

- Open access: articles freely available online

- High visibility within the field

Retaining the copyright to your article

Submit your next manuscript at springeropen.com 